\title{
Complex phenological responses to climate warming trends? Lessons from history
}

\author{
Tim H. SPARKS ${ }^{1}$, KERSTIN HUBER ${ }^{2}$ and RogER L.H. DENNIS ${ }^{1}$ \\ ${ }^{1}$ NERC Centre for Ecology and Hydrology, Monks Wood, Abbots Ripton, Huntingdon, Cambridgeshire PE28 2LS, UK; \\ e-mail: ths@ceh.ac.uk \\ ${ }^{2}$ Fachhochschule München, Lothstrasse 34, 80335 München, Germany
}

Key words. Climate change, climate impacts, Lepidoptera, temperature response, historic data

\begin{abstract}
Responsiveness of Lepidoptera phenology to climate has been detected in a number of species during the current trend in global warming. There is still a question of whether climate signals would be evident in historical data. In this paper we examine the climatic response of 155 species of moths and butterflies collected during the period 1866-1884 in Wiltshire, southern England. In general, species responded to increased temperature in the previous October by delayed appearance and to increased temperature in the current spring by advanced appearance. Thus, differential changes in temperatures of the autumn and spring could well affect changes in the relative pattern of the phenology of species. Attributes influencing the species' ecology were examined to see if they influenced temperature responsiveness. In general, few consistent effects emerged, though responsiveness to climate was found to be greater for species eclosing later in the year, specifically to the previous autumn temperatures, and to hibernal environment, increasingly for species less exposed to air temperatures. These findings warn against expecting simple responses to climate warming.
\end{abstract}

\section{INTRODUCTION}

Historical data can provide valuable evidence on the effects of temperature and other climate variables on the phenology of species, both plant and animal. For some species that have no current phenological monitoring, examination of past data is the only way we can understand the likely effects of climate change on the timing of their life cycles. Furthermore, historic data were collected at an enormous cost in time and money and it is highly desirable that these data are fully exploited.

Data on the phenology of Lepidoptera is largely restricted to butterflies (e.g. Roy \& Sparks, 2000; Forister \& Shapiro, 2003; Stefanescu et al., 2003; Dell et al., 2005) whilst that for moths is sparser (e.g. Kuchlein \& Ellis, 1997; Burton \& Sparks, 2003). These papers broadly suggest a recent advance in the appearance of adult Lepidoptera in conjunction with increased temperatures, and typically a greater advance in species appearing early in the year (e.g. Burton \& Sparks, 2003). Kuchlein \& Ellis (1997) reported on the changing phenology of 104 micromoths in the Netherlands and Burton \& Sparks (2003) on 18 macromoths in southern Germany. Other studies have focussed on the phenology of single pest species such as gypsy moth Lymantria dispar (e.g. Regniere \& Nealis, 2002) or codling moth Cydia pomonella (e.g. Boivin et al., 2005). Only one study has examined the phenological response of a large number of macromoths to temperature and other climatic variables. Woiwod (1997) studied 94 flight periods covering 58 moth species recorded by Rothamsted Insect Survey light traps having more than 20 individuals per year. Phenology was measured by calculating the date for five percentiles of individuals caught $\left(5^{\text {th }}, 25^{\text {th }}, 50^{\text {th }}, 75^{\text {th }}, 95^{\text {th }}\right) ; 93$ were significant, 88 negative (earlier) and 5 positive (later). All percentiles showed a significant tendency, based on the sign test, for advanced emergences. This result confirmed earlier work by Zhou et al. (1996) on aphid phenology using a similar technique. These data relate to the systematic current worldwide period of warming temperatures (Dennis, 1993; IPCC, 2001), experienced regionally in Britain as at Rothamsted (Woiwod, 1997); there are no observations, as far as we are aware, of climate signals in Lepidoptera phenology from large numbers of historic moth and butterfly data.

In this paper, we focus on the susceptibility of British moths and butterflies to climate change in an historical dataset preceding currently recognised warming trends. It is expected that conditions prior to emergence of adults will affect their timing of emergence. For instance, it is expected that higher temperatures and increased sunshine will advance emergence dates but that increased rainfall and cloud may retard emergence dates. An important issue is how far in advance of emergence dates are significant influences recorded; do climate influences impact on the period immediately prior to emergence, a month beforehand when Lepidoptera are passing through the final (pupal) stage, or are influences recorded for earlier periods, and therefore stages, as is the case with population numbers (Pollard \& Yates, 1993)? Any responsiveness of phenology to climate is expected to be complicated by Lepidoptera biology: by the typical period of emergence (i.e. spring to autumn), voltinism or brood number each year, body size, hibernation stage and micro-environment, larval feeding environment (i.e. whether on low growing herbs or higher up on shrubs and trees) internally or externally on substrates. Susceptibility may also be related to edge of range, conservation status, migration capacity and ease of recording or recording 


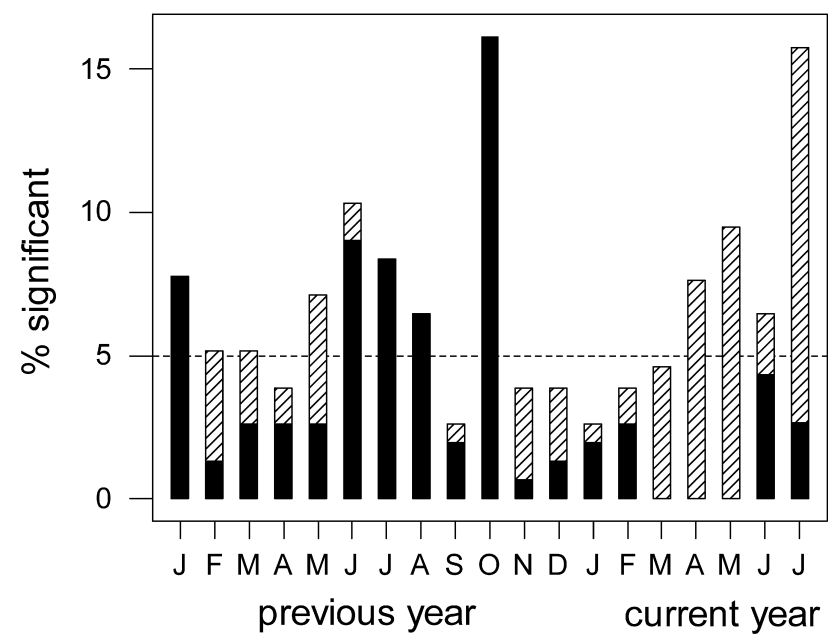

Fig. 1. Percentage of significant positive (solid bars) and negative (hatched bars) correlations between first appearance date of lepidopteran species and calendar monthly mean temperatures. The dotted line represents the $5 \%$ level of significant results expected by chance.

methodology, i.e. whether day flying or night flying. In addition, indirect effects via host plant phenology may be important, such as plant development following previous year's rainfall, although beyond the scope of the current work.

Work on competitive, stress-tolerant and ruderal (CSR) strategies of larval host plants among British butterflies would suggest that responsiveness to climate changes is not unexpected (Dennis et al., 2004). As such, it is clearly of value to determine whether susceptibility to climate change links to biological attributes in moths and butterflies. The present dataset allows some links to be directly tested.

In this paper we examine the phenology of a large number of Lepidoptera species recorded in the second half of the nineteenth century, examine their responsiveness to temperature, and seek to answer the following questions:

- How far in advance of emergence do climate influences affect emergence dates?

- What aspects of a species' ecology influences its responsiveness to temperature [for example, later flying species may actually emerge later with increasing temperatures (Buse \& Good, 1996; Woiwod, 1997)]?

\section{MATERIAL AND METHODS}

The Marlborough College Natural History Society (MCNHS) $\left(51^{\circ} 25^{\prime} \mathrm{N}, 1^{\circ} 44^{\prime} \mathrm{W}\right)$ collected a vast amount of phenological data in the mid-nineteenth century and in 1885 privately published the first 19 years of its results. In this paper we focus on the first observations of appearance in 1866-1884 of Lepidoptera (moths and butterflies) because the phenology of moths, in particular, is rarely studied. Over 500 species of Lepidoptera are included in the MCNHS report. However, we have focussed on the 155 species (121 moths and 34 butterflies; Table 1) for which at least 10 years of data were present. We do not know exactly how these records were obtained but assume they were collected by mem-

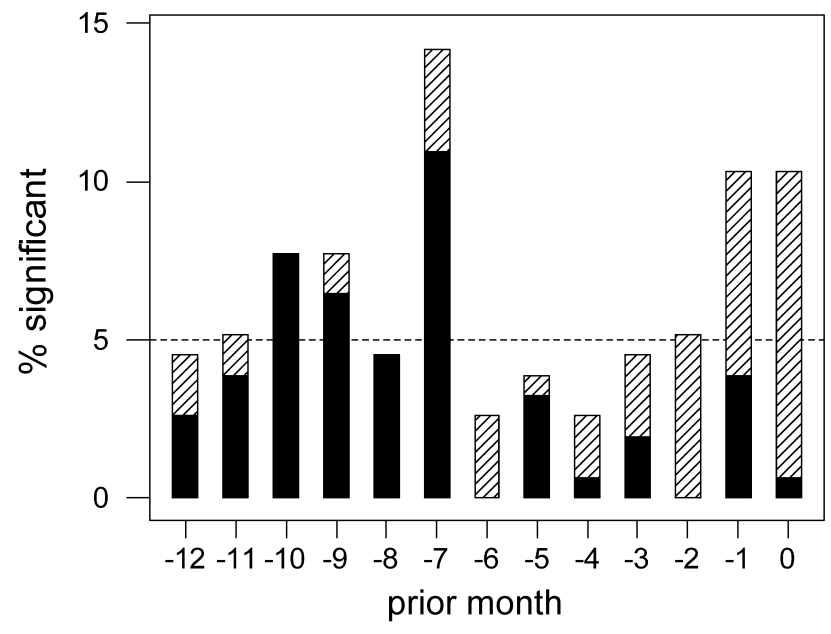

Fig. 2. As Fig. 1 except summarised by month prior to the mean date of each species.

bers of the MCNHS with verification by TA Preston, founder of the MCNHS.

In the same report are summarised weather measurements taken at Marlborough. From these records we have abstracted monthly averages of mean air temperature and total rainfall and have converted these to metric scales.

In determining the impact of climate, we examined which months' temperatures and rainfall totals were most influential on first appearance patterns. We had anticipated that conditions during the latter stages of larval growth and pupal development would most likely affect development rates and timing of adult stages (Dell et al., 2005). For each species, we examined the correlation with the weather in the month in which its mean first appearance date occurred, and in the preceding months back to January of the previous year. Correlations were summarised in two ways, by calendar month and by month prior to the mean date of each species. The pattern of significant correlations with calendar monthly temperatures (Fig. 1) was similar to that of mean correlation coefficients. Significant correlations in the current year were predominantly negative $($ warmer $=$ earlier $)$ while those of the previous year, particularly October, were predominantly positive. The pattern of correlations with prior months was much clearer (Fig. 2); those with recent months were negative but were positive further back in time. Significant correlations with monthly rainfalls (Fig. 3) were rarely above the level expected by chance alone, but tended to be positive (higher rainfall = later). The pattern with prior months' rainfall was broadly similar (not shown). Because significant rainfall correlations were rarely more than the expected background levels, rainfall was subsequently disregarded in favour of temperature. Figs 1, 2 and 4 show that two periods of temperature appear to be important in determining first appearance date: October of the previous year and the three prior months to mean date. Subsequently a multiple regression for each species of first appearance date on October temperature and on the mean of the previous three monthly mean air temperatures was undertaken to predict the likely effect of a $1{ }^{\circ} \mathrm{C}$ increase in temperature in these two periods. The regression coefficients from these two variables (temperature responses) for the 155 species were examined in relation to different aspects of species ecology using either weighted regression, weighted ANOVA or weighted ANCOVA as appropriate with weights equal to the numbers of years of records for each species. Thus, greater emphasis was given to species whose temperature response was 
TABLE 1. Summary information on the 34 butterfly and 121 moth species for which at least ten years of data are available. Species are arranged in increasing date of mean first appearance in each group.

\begin{tabular}{|c|c|c|c|c|c|}
\hline Scientific name & English name & mean day & mean date & sd & $\mathrm{n}$ \\
\hline \multicolumn{6}{|l|}{ BUTTERFLIES } \\
\hline Aglais urticae & Small Tortoiseshell & 58.3 & $27 \mathrm{Feb}$ & 18.7 & 16 \\
\hline Gonepteryx rhamni & Brimstone & 62.0 & $03 \mathrm{Mar}$ & 13.8 & 17 \\
\hline Inachis io & Peacock & 84.9 & $26 \mathrm{Mar}$ & 37.8 & 16 \\
\hline Nymphalis polychloros & Large Tortoiseshell & 98.7 & 09 Apr & 17.1 & 12 \\
\hline Pieris rapae & Small White & 99.9 & $10 \mathrm{Apr}$ & 17.5 & 18 \\
\hline Pieris napi & Green-veined White & 111.9 & $22 \mathrm{Apr}$ & 19.0 & 18 \\
\hline Pieris brassicae & Large White & 116.2 & $26 \mathrm{Apr}$ & 23.9 & 18 \\
\hline Vanessa atalanta & Red Admiral & 120.4 & $30 \mathrm{Apr}$ & 35.9 & 12 \\
\hline Anthocharis cardamines & Orange Tip & 127.6 & 08 May & 11.6 & 18 \\
\hline Pyrgus malvae & Grizzled Skipper & 134.6 & 15 May & 8.7 & 18 \\
\hline Lycaena phlaeas & Small Copper & 136.2 & 16 May & 11.5 & 17 \\
\hline Erynnis tages & Dingy Skipper & 136.4 & 16 May & 8.0 & 18 \\
\hline Pararge aegeria & Speckled Wood & 138.8 & 19 May & 22.9 & 17 \\
\hline Lasiommata megera & Wall Brown & 139.1 & 19 May & 10.5 & 17 \\
\hline Coenonympha pamphilus & Small Heath & 140.2 & 20 May & 9.4 & 18 \\
\hline Boloria euphrosyne & Pearl-bordered Fritillary & 140.9 & 21 May & 10.4 & 18 \\
\hline Callophrys rubi & Green Hairstreak & 145.1 & 25 May & 13.0 & 14 \\
\hline Vanessa cardui & Painted Lady & 146.0 & 26 May & 23.5 & 16 \\
\hline Polyommatus icarus & Common Blue & 149.5 & 30 May & 20.9 & 19 \\
\hline Hamearis lucina & Duke of Burgundy Fritillary & 150.0 & 30 May & 9.3 & 17 \\
\hline Boloria selene & Small Pearl-bordered Fritillary & 154.3 & 03 Jun & 12.9 & 17 \\
\hline Aricia agestis & Brown Argus & 154.8 & 04 Jun & 13.5 & 13 \\
\hline Ochlodes venata & Large Skipper & 157.0 & 06 Jun & 11.5 & 17 \\
\hline Cupido minimus & Small Blue & 163.1 & 12 Jun & 19.3 & 16 \\
\hline Maniola jurtina & Meadow Brown & 168.1 & 17 Jun & 19.4 & 17 \\
\hline Aphantopus hyperantus & Ringlet & 182.5 & 01 Jul & 6.1 & 13 \\
\hline Argynnis aglaja & Dark Green Fritillary & 184.2 & $03 \mathrm{Jul}$ & 19.6 & 15 \\
\hline Pyronia tithonus & Gatekeeper & 187.7 & $07 \mathrm{Jul}$ & 20.9 & 10 \\
\hline Thymelicus sylvestris & Small Skipper & 191.4 & $10 \mathrm{Jul}$ & 10.4 & 15 \\
\hline Argynnis adippe & High Brown Fritillary & 192.1 & $11 \mathrm{Jul}$ & 16.7 & 14 \\
\hline Melanargia galathea & Marbled White & 194.7 & $14 \mathrm{Jul}$ & 13.7 & 14 \\
\hline Argynnis paphia & Silver-washed Fritillary & 198.3 & $17 \mathrm{Jul}$ & 14.1 & 14 \\
\hline Polyommatus coridon & Chalkhill Blue & 203.2 & $22 \mathrm{Jul}$ & 16.0 & 10 \\
\hline Neozephyrus quercus & Purple Hairstreak & 209.3 & $28 \mathrm{Jul}$ & 18.0 & 12 \\
\hline \multicolumn{6}{|l|}{ Мотнs } \\
\hline Theria rupicapraria & Early Moth & 48.1 & $17 \mathrm{Feb}$ & 15.6 & 15 \\
\hline Agriopis marginaria & Dotted Border & 65.6 & 07 Mar & 14.4 & 15 \\
\hline Alsophila aescularia & March Moth & 75.0 & $16 \mathrm{Mar}$ & 12.9 & 10 \\
\hline Scoliopteryx libatrix & The Herald & 78.1 & 19 Mar & 41.5 & 11 \\
\hline Orthosia cerasi & Common Quaker & 80.6 & $22 \mathrm{Mar}$ & 13.9 & 12 \\
\hline Orthosia incerta & Clouded Drab & 86.9 & $28 \mathrm{Mar}$ & 11.7 & 10 \\
\hline Orthosia gothica & Hebrew Character & 87.8 & $29 \mathrm{Mar}$ & 23.7 & 14 \\
\hline Archiearis parthenias & Orange Underwing & 90.2 & 31 Mar & 6.9 & 10 \\
\hline Selenia lunularia & Lunar Thorn & 96.8 & 07 Apr & 18.7 & 12 \\
\hline Xanthorhoe fluctuata & Garden Carpet & 116.4 & $26 \mathrm{Apr}$ & 13.9 & 17 \\
\hline Anticlea derivata & The Streamer & 125.1 & 05 May & 11.3 & 10 \\
\hline Eupithecia vulgata & Common Pug & 130.0 & 10 May & 29.1 & 12 \\
\hline Menophra abruptaria & Waved Umber & 130.2 & 10 May & 10.9 & 13 \\
\hline Xanthorhoe spadicearia & Red Twin-spot Carpet & 130.3 & 10 May & 6.8 & 14 \\
\hline Opisthograptis luteolata & Brimstone Moth & 133.2 & 13 May & 11.2 & 17 \\
\hline Xanthorhoe biriviata & Balsam Carpet & 133.7 & 14 May & 18.8 & 15 \\
\hline Watsonella cultraria & Barred Hook-tip & 138.4 & 18 May & 26.7 & 11 \\
\hline Mimas tiliae & Lime Hawk-moth & 138.6 & 19 May & 27.3 & 12 \\
\hline Spilosoma lubricipeda & White Ermine & 140.1 & 20 May & 10.2 & 17 \\
\hline Phragmatobia fuliginosa & Ruby Tiger & 140.5 & 20 May & 30.1 & 10 \\
\hline Petrophora chlorosata & Brown Silver-line & 141.1 & 21 May & 13.3 & 16 \\
\hline Asthena albulata & Small White Wave & 141.4 & 21 May & 9.7 & 14 \\
\hline Lomographa bimaculata & White-pinion Spotted & 142.3 & 22 May & 16.8 & 11 \\
\hline Jodis lactearia & Little Emerald & 142.4 & 22 May & 7.7 & 17 \\
\hline Xanthorhoe montanata & Silver-ground Carpet & 142.5 & 23 May & 8.7 & 15 \\
\hline Cabera pusaria & Common White Wave & 142.7 & 23 May & 8.9 & 16 \\
\hline Lomographa temerata & Clouded Silver & 143.7 & 24 May & 18.1 & 11 \\
\hline Eupithecia subfuscata & Grey Pug & 144.9 & 25 May & 16.2 & 11 \\
\hline Anthophila fabriciana & Nettle-tap Moth & 145.8 & 26 May & 37.0 & 12 \\
\hline Diaphora mendica & Muslin Moth & 145.8 & 26 May & 14.8 & 13 \\
\hline Chiasmia clathrata & Latticed Heath & 146.9 & 27 May & 31.6 & 17 \\
\hline Callistege mi & Mother Shipton & 149.0 & 29 May & 13.1 & 15 \\
\hline Spilosoma lutea & Buff Ermine & 149.1 & 29 May & 9.8 & 17 \\
\hline Odontopera bidentata & Scalloped Hazel & 149.2 & 29 May & 12.7 & 10 \\
\hline Electrophaes corylata & Broken-barred Carpet & 149.7 & 30 May & 12.2 & 15 \\
\hline Lomaspilis marginata & Clouded Border & 149.7 & 30 May & 13.1 & 18 \\
\hline Phytometra viridaria & Small Purple-barred & 149.8 & 30 May & 16.2 & 13 \\
\hline Tyria jacobaeae & The Cinnabar & 150.1 & 30 May & 13.9 & 16 \\
\hline Phlogophora meticulosa & Angle Shades & 150.2 & 30 May & 20.5 & 13 \\
\hline Scopula floslactata & Cream Wave & 150.7 & 31 May & 13.4 & 12 \\
\hline Korscheltellus lupulina & Common Swift & 151.7 & 01 Jun & 12.6 & 15 \\
\hline
\end{tabular}


Chloroclysta siterata

Cyclophora linearia

Macroglossum stellatarum

Drepana falcataria

Pechipogo strigilata

Mamestra brassicae

Crambus pratella

Ligdia adustata

Autographa gamma

Calliteara pudibunda

Parasemia plantaginis

Minoa murinata

Cosmorhoe ocellata

Odezia atrata

Cilix glaucata

Cyclophora punctaria

Acronicta psi

Cabera exanthemato

Ptilodon capucina

Adscita geryon

Opsibotys fuscalis

Charanyca trigrammica

Ematurga atomaria

Atolmis rubricollis

Smerinthus ocellata

Camptogramma bilineata

Noctua pronuba

Sphinx ligustri

Deilephila porcellus

Apamea sordens

Laothoe populi

Eurrhypara hortulata

Udea olivalis

Epirrhoe rivata

Agrotis exclamationis

Hada plebeja

Chloroclysta truncata

Lacanobia oleracea

Hydrelia flammeolaria

Oligia strigilis

Idaea aversata

Deilephila elpenor

Hepialus humul

Scotopteryx luridata

Hypena proboscidalis

Idaea seriata

Rusina ferruginea

Zygaena filipendulae

Apamea monoglypha

Euphyia unangulata

Diarsia mendica

Udea prunalis

Perizoma alchemillata

Rhinoprora rectangulata

Euthrix potatoria

Campaea margaritata

Cidaria fulvata

Perizoma didymata

Diachrysia chrysitis

Aphomia sociella

Amphipoea oculea

Abraxas grossulariata

Mythimna pallens

Chloroclysta citrata

Philereme vetulata

Eulithis pyraliata

Xestia c-nigrum

Arctia caja

Triodia sylvina

Peribatodes rhomboidaria

Cryphia domestica

Macaria wauaria

Paradrina clavipalpis

Ourapteryx sambucaria

Scotopteryx chenopodiata

Hydriomena furcata

Tholera decimalis

Ennomos alniaria

Orgyia antiqua

Diloba caeruleocephala
Red-green Carpet

Clay Triple-lines

Humming-bird Hawk-moth

Pebble Hook-tip

Common Fan-foot

Cabbage Moth

NONE

Scorched Carpet

Silver Y

Pale Tussock

Wood Tiger

Drab Looper

Purple Bar

Chimney Sweeper

Chinese Character

Maiden's Blush

Grey Dagger

Common Wave

Coxcomb Prominent

Cistus Forester

NONE

Treble Lines

Common Heath

Red-necked Footman

Eyed Hawk-moth

Yellow Shell

Large Yellow Underwing

Privet Hawk-moth

Small Elephant Hawk-moth

Rustic Shoulder-knot

Poplar Hawk-moth

Small Magpie

NONE

Wood Carpet

Heart and Dart

The Shears

Common Marbled Carpet

Bright-line Brown-eye

Small Yellow Wave

Marbled Minor

Riband Wave

Elephant Hawk-moth

Ghost Moth

July Belle

The Snout

Small Dusty Wave

Brown Rustic

Six-spot Burnet

Dark Arches

Sharp-angled Carpet

Ingrailed Clay

NONE

Small Rivulet

Green Pug

The Drinker

Light Emerald

Barred Yellow

Twin-spot Carpet

Burnished Brass

Bee Moth

Ear Moth

The Magpie

Common Wainscot

Dark Marbled Carpet

Brown Scallop

Barred Straw

Setaceous Hebrew Character

Garden Tiger

Orange Swift

Willow Beauty

Marbled Beauty

The V-Moth

Pale Mottled Willow

Swallow-tailed Moth

Shaded Broad-bar

July Highflyer

Feathered Gothic

Canary-shouldered Thorn

The Vapourer

Figure of Eigh 


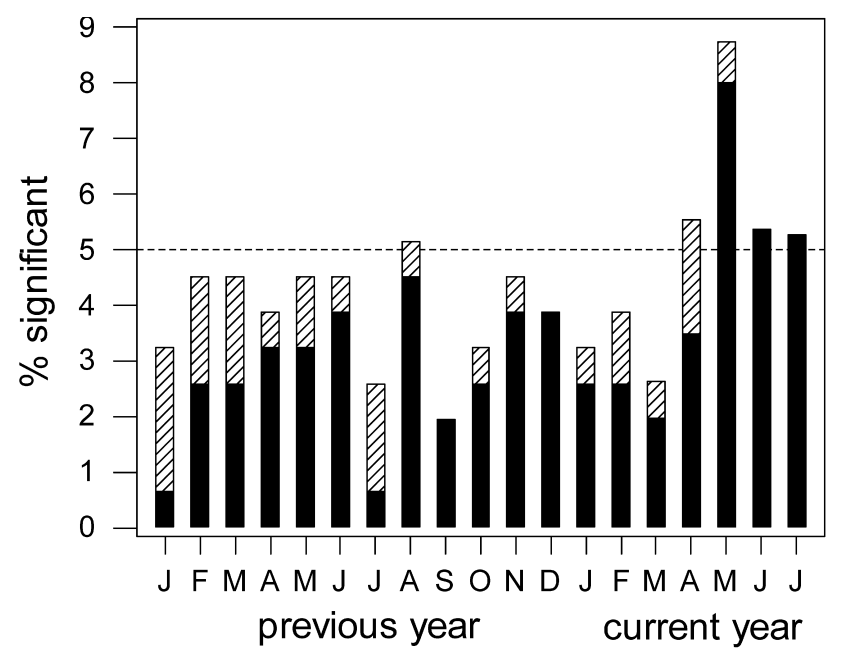

Fig. 3. Percentage of significant positive (solid bars) and negative (hatched bars) correlations between first appearance date and calendar monthly rainfall. The dotted line represents the $5 \%$ level of significant results expected by chance.

derived from more years of data. Mean values are presented \pm se, regression, ANOVA and ANCOVA results are summarised by $\mathrm{F}$ ratio and probability value.

Information on the different aspects of the species ecology were abstracted and converted to scales and categories as shown in Table 2.

\section{RESULTS}

Table 1 lists the species, the mean and standard deviation of first appearance date and the numbers of years of data available. Two species had mean first appearance date in February, nine in March, seven in April, 42 in May, 57 in June, 34 in July, three in August and one in September. Butterfly species were significantly earlier, on average, than moth species (May 26 and June 10 respectively, $\left.\mathrm{F}_{1,153}=6.62 \mathrm{P}=0.011\right)$ and significantly better recorded, respectively an average of 15.6 years of data compared to 12.9 years $\left(\mathrm{F}_{1,153}=37.75 \mathrm{P}<0.001\right)$. The standard deviation of butterflies was slightly but not significantly smaller than that of moths (16.3 days and 18.1 days respectively, $\left.F_{1,153}=1.43 \mathrm{P}=0.23\right)$. Neither the slightly negative relationship $\left(\mathrm{F}_{1,32}=2.32, \mathrm{P}=0.14\right)$ between standard deviation and mean date for butterflies

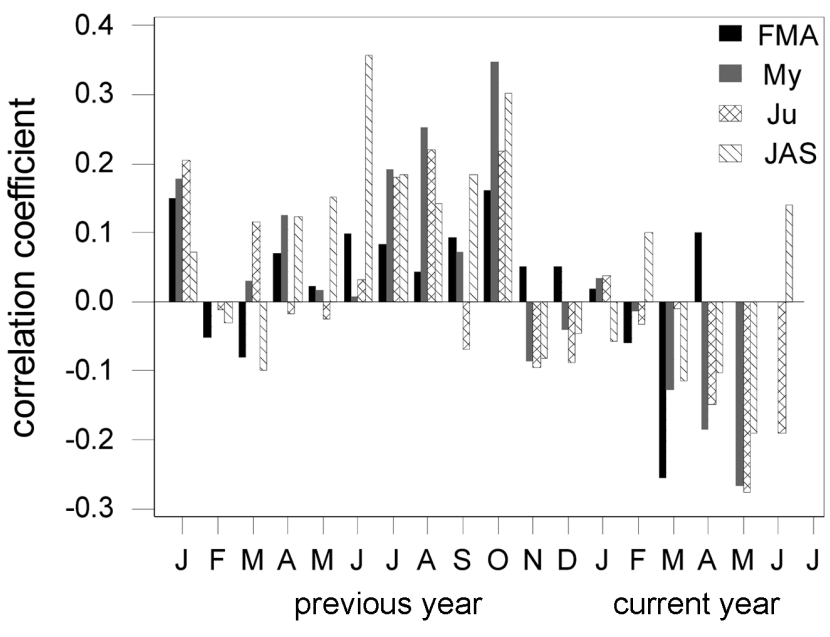

Fig. 4. Mean correlation coefficients with monthly mean temperatures grouped by four first appearance periods (February-April, May, June, July-September).

or the slightly positive relationship $\left(\mathrm{F}_{1,119}=2.45, \mathrm{P}=\right.$ 0.089 ) for moths was significant.

There were no significant trends in temperature during the study period (each month, $\mathrm{P}>0.10$ ). The response to October temperature averaged $3.63 \pm 0.38$ days $/{ }^{\circ} \mathrm{C}$ i.e. warmer Octobers tended to delay first appearance date in the following year. The response to three prior months' temperature, in contrast, averaged $-3.05 \pm 0.54$ days $/{ }^{\circ} \mathrm{C}$ indicating earlier first appearance with warmer temperatures preceding first appearance. Fig. 5 presents histograms of the two responses clearly indicating the contrasting effects. An attempt has been made to ascertain whether the response to climate of the species falls into any natural or logical groupings.

\section{Conservation status}

The responsiveness of species to October and three prior months temperatures did not differ significantly between species of conservation interest $(n=126)$ and other species $(n=29$; Table 3$)$. Whilst species of conservation status did not differ significantly from other species in mean date of appearance, they were significantly larger $(40.0 \pm 1.3 \mathrm{~mm}$ and $33.0 \pm 1.9 \mathrm{~mm}$ respectively, $\left.\mathrm{F}_{1,153}=5.45 \mathrm{P}=0.021\right)$.

TABLE 2. A summary of the investigated variables.

\begin{tabular}{lll}
\hline Variable & Source & Reduced to \\
\hline Conservation status & Waring et al. (2003) & Binary: conservation interest or not \\
Distributional range & Emmet \& Heath (1991) & Binary: England \& Wales or including Scotland \\
Voltinism & Emmet \& Heath (1991) & Binary: mainly univoltine or more generations \\
Day or night flying & Emmet \& Heath (1991) & Binary: predominantly day or night flying \\
Wing expanse & Emmet \& Heath (1991), Waring et al. (2003) & Average of extremes. \\
Feeding preferences & Emmet \& Heath (1991) & Binary: Grasses/herbs or shrubs/trees \\
Migratory status & Emmet \& Heath (1991) & Binary: partly/wholly migratory or resident \\
Hibernation environment & Emmet \& Heath (1991), Waring et al. (2003) & 5 point ordinal scale 1 = exposed, 5 = underground \\
Larval exposure & Emmet \& Heath (1991) & 3 point ordinal scale: not, moderately or fully exposed \\
Pupal exposure & Emmet \& Heath (1991) & 3 point ordinal scale: not, moderately or fully exposed \\
\hline
\end{tabular}



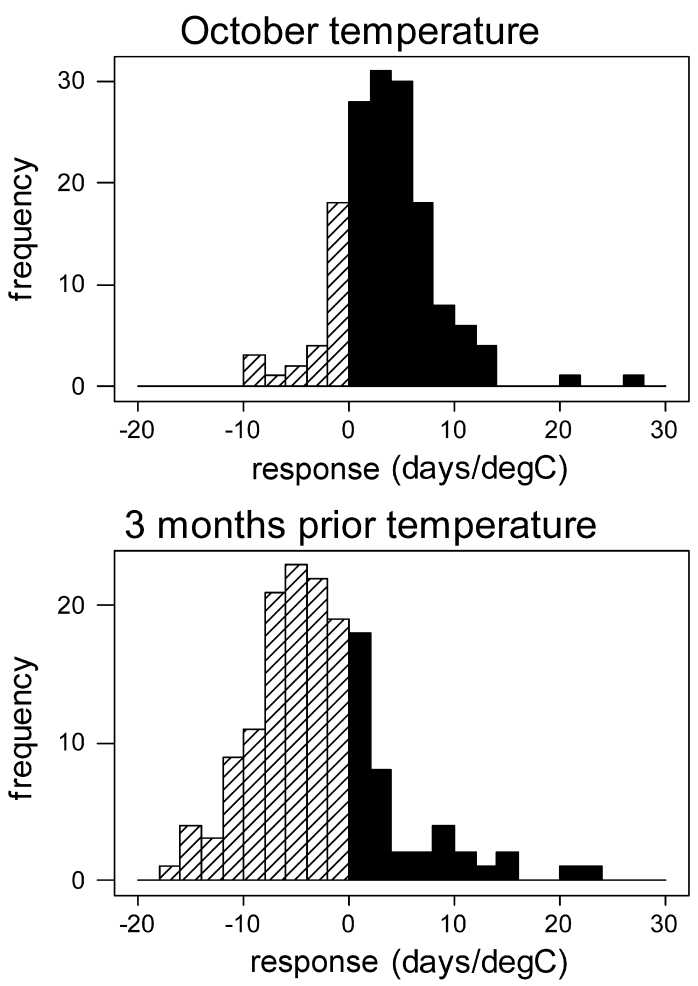

Fig. 5. Histograms of responses to a $1{ }^{\circ} \mathrm{C}$ increase in October and three prior months temperature. Hatched bars negative response, solid bars positive response.

\section{Distributional range}

The responsiveness of species with ranges including Scotland was not significantly different from those restricted to England and Wales (Table 3).

\section{Voltinism}

The responsiveness of univoltine species for three prior months $\left(-3.74 \pm 0.58\right.$ days $\left./{ }^{\circ} \mathrm{C}, \mathrm{n}=116\right)$ was greater, but not significantly greater (Table 3 ) than multivoltine species $\left(-1.22 \pm 1.23\right.$ days $\left./{ }^{\circ} \mathrm{C}, \mathrm{n}=36\right)$.

\section{Mean date of appearance}

A significant linear relationship existed between October response and mean first appearance date but not for the three prior months (Table 3). For October temperatures, the delayed first appearance with warmer temperatures was more pronounced for species first observed later in the year. When examined between four month groups (February-April, May, June, July-September) significant differences between month groups were apparent for both temperature responses (Table 3, Fig. 6). In Fig. 6 the clear trend for a greater positive response to October temperatures and lower response to three prior months' temperature is apparent.

\section{Butterfly or moth}

No significant differences were found in responsiveness of butterflies and moths (Table 3).

\section{Day or night flying}

Day flying Lepidoptera (mean $2.57 \pm 0.56$ days $/{ }^{\circ} \mathrm{C}, \mathrm{n}=$ 44) were almost significantly less responsive to October

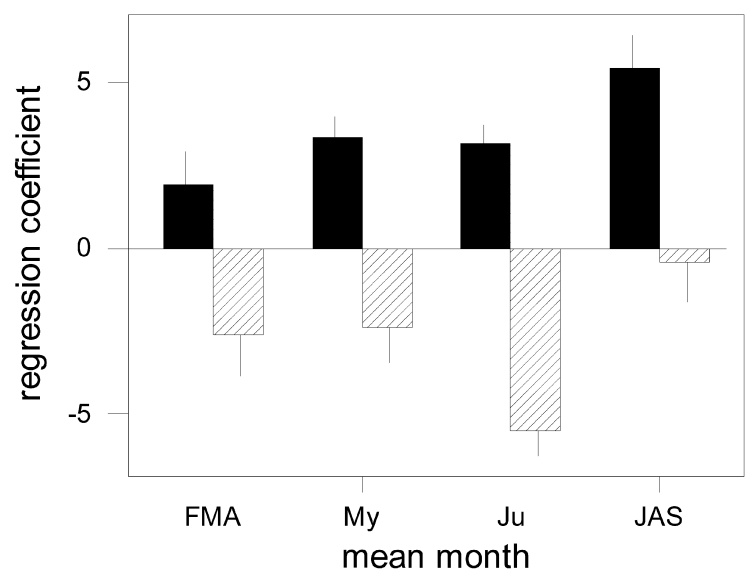

Fig. 6. Mean regression coefficients of first appearance date on October temperature (solid bars) and three prior months temperatures (hatched bars), grouped by four first appearance periods (February-April, May, June, July-September). Vertical lines represent one standard error of the mean.

temperature than night flying Lepidoptera $(4.06 \pm 0.48$ days $/{ }^{\circ} \mathrm{C}, \mathrm{n}=111$, Table 3 ). Day flying Lepidoptera were on average earlier (May $29 \pm 6$ and June $10 \pm 3$, respectively, $\left.\mathrm{F}_{1,153}=4.36 \mathrm{P}=0.038\right)$ and larger than night flying Lepidoptera $(43.2 \pm 2.3 \mathrm{~mm}$ and $36.9 \pm 1.3 \mathrm{~mm}$ respectively, $F_{1,153}=7.01 \mathrm{P}=0.009$ ). However, after elimination of mean date and size in an ANCOVA, the difference in October response was further from significance $\left(\mathrm{F}_{1,151}=\right.$ $1.59, \mathrm{P}=0.21$ ).

\section{Wing size}

There was no significant relationship between wing expanse and responsiveness (Table 3).

\section{Family}

Table 4 summarises the mean responses of the eight families for which at least five members were represented. Despite the large differences, there was no significant difference in responsiveness between families (Table 3 ) as a consequence of the large variability within families.

\section{Feeding preferences}

Species feeding on grasses and herbs had a mean temperature response to three prior months temperature of $-3.62 \pm 0.71$ days $/{ }^{\circ} \mathrm{C}(\mathrm{n}=89)$ compared to shrub and tree feeders whose mean response was $-1.56 \pm 0.90$ days $/{ }^{\circ} \mathrm{C}$ $(\mathrm{n}=53)$. The difference was not quite significant (Table 3).

\section{Migrants}

The few migrant or partially migrant species were significantly less temperature responsive to October temperatures $\left(1.17 \pm 1.19\right.$ days $\left./{ }^{\circ} \mathrm{C}, \mathrm{n}=14\right)$ than residents (3.88 \pm 0.40 days $\left./{ }^{\circ} \mathrm{C}, \mathrm{n}=141\right)$ (Table 3). However migrants were significantly earlier (May $8 \pm 10 \mathrm{cf}$. June 9 $\left.\pm 3, \mathrm{~F}_{1,153}=13.59 \mathrm{P}<0.001\right)$ and larger $(53.4 \pm 3.4 \mathrm{~mm}$ cf. $\left.37.2 \pm 1.2, \mathrm{~F}_{1,153}=21.33 \mathrm{P}<0.001\right)$. After eliminating the effects of mean date and size in ANCOVA, there was 
TABLE 3. Statistical tests of differential responses to October and mean of three prior month temperatures on a range of Lepidoptera attributes. Reg $=$ regression, significant $(\mathrm{P}<0.05)$ results in bold.

\begin{tabular}{|c|c|c|c|c|c|}
\hline \multirow{2}{*}{ Variable } & \multirow{2}{*}{ Test } & \multicolumn{2}{|c|}{ Oct coefficient } & \multicolumn{2}{|c|}{ Three prior months coefficient } \\
\hline & & Test statistic & $\mathrm{P}$ & Test statistics & $\mathrm{P}$ \\
\hline Conservation status & ANOVA & $F_{1,153}=0.46$ & 0.50 & $\mathrm{~F}_{1,153}=0.15$ & 0.70 \\
\hline Distributional range & ANOVA & $\mathrm{F}_{1,153}=0.26$ & 0.61 & $\mathrm{~F}_{1,153}=0.10$ & 0.75 \\
\hline Voltinism & ANOVA & $\mathrm{F}_{1,150}=1.51$ & 0.22 & $\mathrm{~F}_{1,150}=2.94$ & 0.089 \\
\hline Mean appearance date & Reg & $F_{1,153}=8.30$ & 0.005 & $\mathrm{~F}_{1,153}=0.42$ & 0.52 \\
\hline Appearance month group & ANOVA & $F_{3,151}=3.25$ & 0.024 & $F_{3,151}=4.83$ & 0.003 \\
\hline Butterfly vs Moth & ANOVA & $\mathrm{F}_{1,153}=2.41$ & 0.12 & $\mathrm{~F}_{1,153}=0.75$ & 0.39 \\
\hline Day vs night flying & ANOVA & $\mathrm{F}_{1,153}=3.76$ & 0.054 & $\mathrm{~F}_{1,153}=0.59$ & 0.44 \\
\hline Wing expanse & Reg & $\mathrm{F}_{1,153}=2.37$ & 0.13 & $\mathrm{~F}_{1,153}=0.43$ & 0.51 \\
\hline Family & ANOVA & $\mathrm{F}_{7,131}=0.91$ & 0.50 & $\mathrm{~F}_{7,131}=0.84$ & 0.55 \\
\hline Feeding preferences & ANOVA & $\mathrm{F}_{1,140}=0.97$ & 0.33 & $\mathrm{~F}_{1,140}=3.20$ & 0.076 \\
\hline Migratory status & ANOVA & $F_{1,153}=5.82$ & 0.017 & $\mathrm{~F}_{1,153}=2.35$ & 0.13 \\
\hline Hibernation environment & Reg & $\mathrm{F}_{1,153}=1.22$ & 0.27 & $F_{1,153}=4.95$ & 0.028 \\
\hline Larval exposure & Reg & $\mathrm{F}_{1,136}=0.76$ & 0.38 & $\mathrm{~F}_{1,136}=0.58$ & 0.45 \\
\hline Pupal exposure & Reg & $F_{1,136}=0.66$ & 0.42 & $\mathrm{~F}_{1,136}=0.01$ & 0.91 \\
\hline
\end{tabular}

still a difference in temperature responsiveness $\left(\mathrm{F}_{1,151}=\right.$ $5.40 \mathrm{P}=0.021)$.

\section{Hibernation environment}

Nine species that overwinter as adults were eliminated from this test. A significant relationship existed between three prior month temperature response and hibernation environment (Table 3). Species whose hibernation stages were less exposed had a greater negative response to temperature.

\section{Larval and pupal exposure}

No significant differences in responsiveness were detected (Table 3).

\section{DISCUSSION}

Since the phenology of moths has been rarely reported, and then only for the period affected by systematic changes in temperatures over the past half century (Woiwod, 1997), this use of historical data is valuable in increasing our understanding of the effects of climate on life cycle timings. During the period of study, annual

TABLE 4. Mean \pm se for response of Lepidoptera families represented by at least five species.

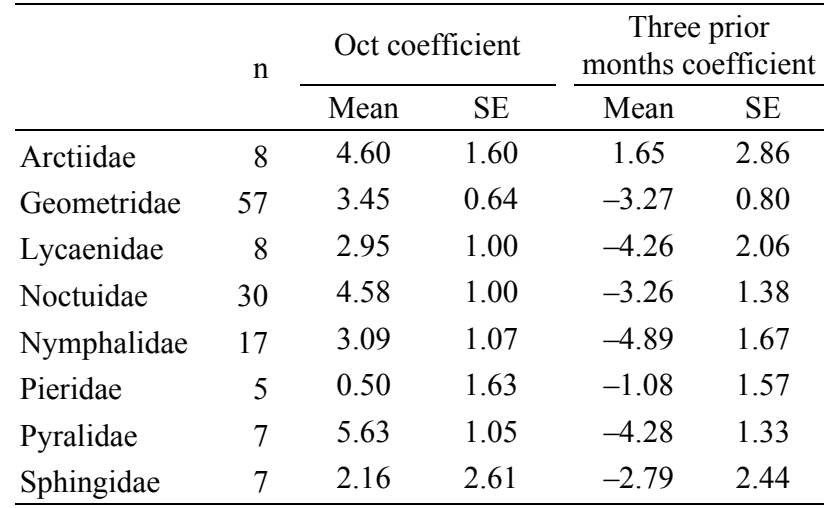

mean temperatures were c. $0.9^{\circ} \mathrm{C}$ cooler than currently. The Lepidoptera include species of high conservation status and high pest status, and species of both low and high (migratory) mobility. As such they are an interesting group with which to further the study of climate impacts. What is very obvious is that, taken as a whole, these species are generally responsive to a warming environment. In general, species appeared much more responsive to temperature than rainfall, although this may not be true in other countries where water is deficient or in Britain during periods with a wider range of rainfall conditions. Other factors, such as host abundance or population dynamics may play a role in first detection date (for an example with birds see Tryjanowski et al., 2005) but we do not have contemporary data on these for our Lepidoptera. Temperatures of three months prior to appearance seemed to affect appearance in a negative way; higher temperatures advancing appearance dates. It was somewhat of a surprise that temperatures of the previous autumn, and in particular October, were having such a positive effect on appearance date. There was no significant correlation between temperature in the previous October and those in the current year (each month, $\mathrm{P}>$ 0.13 ) to explain this result. Since the magnitude of change arising from October and three prior months temperatures approximately cancel one another out the relative changes in temperature in these two periods will have a major influence on the changing pattern of phenology.

Some of the responses in individual species may be aberrant because of small sample size, but we used weighted regression to give greater emphasis to results based on more years.

In the examination of a large number of species attributes, we were surprised that we did not detect more patterns in the temperature responses of these Lepidoptera. Migratory species did not appear very responsive to pre- 
vious October temperature; an intuitive result since they are not in Britain at that time to experience that weather. Climate effects on migrants are likely to be very complex as they experience a range of climates at different parts of their life cycle (Sparks et al., 2005). Overall, however, migrants are likely to be more flexible in response to a changing climate than their sedentary cousins (Dennis, 1993).

The greatest difference appeared to result from the timing of the species; late flying species are particularly sensitive to previous October temperature and much less so to three prior months' temperature whilst the response of June species to the latter was particularly strong. If climate warming happens evenly through the year, these results suggest that late flying species will get progressively later whilst June species will get earlier. If change does not occur in synchrony with host or predator phenology then serious consequences may become apparent (for discussion, see Root et al., 2003).

Do recent advances in spring phenology of moths and butterflies point to a differential warming, with greater temperature increase at the beginning of the year and less in autumn? Could recent advances in phenology be retarded as autumn temperatures start to increase? An obvious caveat is that our data are from a relatively small number of years from a single location. Our inability to detect many patterns in examining temperature response to species attributes may be because they are difficult to detect statistically in our relatively short data set or that we did not have available other appropriate species attributes. A more worrying alternative is that temperature responses in Lepidoptera species appear random or chaotic. If so, our ability to predict the consequences of a changing climate on Lepidoptera communities may be seriously challenged.

We hope that the findings in the Marlborough data will encourage other holders of long term data sets to examine them for features of species' ecology that may influence temperature response, in order that the consequences of a changing climate on Lepidoptera can be more fully understood. Influences that emerge in the current dataset (i.e. related to hibernal environment and mean emergence date) suggest complicated scenarios with climate change and this, together with the absence of definitive explanations for these relationships, points to fruitful areas for future research.

ACKNOWLEDGEMENTS. We thank two anonymous referees for their comments on an earlier version of this paper.

\section{REFERENCES}

Boivin T., Chadoeuf J., Bouvier J.C., Beslay D. \& Sauphanor B. 2005: Modelling the interactions between phenology and insecticide resistance genes in the codling moth Cydia pomonella. Pest Manag. Sci. 61: 53-67.
Burton J.F. \& Sparks T.H. 2003: The flight phenological responses of Lepidoptera to climate change in Britain and Germany. Atalanta 34: 3-16.

Buse A. \& Good J.E.G. 1996: Synchronization of larval emergence in winter moth (Operophtera brumata L.) and budburst in pedunculate oak (Quercus robur L.) under simulated climate change. Ecol. Entomol. 21: 335-343.

Dell D., Sparks T.H. \& Dennis R.L.H. 2005: Climate change and the effect of increasing spring temperatures on emergence dates of the flagship butterfly Apatura iris (Lepidoptera: Nymphalidae). Eur. J. Entomol. 102: 161-167.

DenNis R.L.H. 1993: Butterflies and Climate Change. Manchester University Press, Manchester, 302 pp.

Dennis R.L.H., Hodgson J.G., Grenyer R., Shreeve T.G. \& Roy D.B. 2004: Host plants and butterfly biology. Do host plant strategies drive butterfly status? Ecol. Entomol. 29: 12-26.

Eмmet A.M. \& HeAth J. (eds) 1991: The Moths and Butterflies of Great Britain and Ireland. Vol. 7, Part 2. Harley Books, Colchester, $400 \mathrm{pp}$.

Forister M.L. \& SHAPIro A.M. 2003: Climatic trends and advancing spring flight of butterflies in lowland California. Global Change Biol. 9: 1130-1135.

IPCC 2001: Climate Change 2001: The Scientific Basis. Contribution of Working Group I to the Third Assessment Report of the Intergovernmental Panel on Climate Change. Cambridge University Press, Cambridge.

Kuchlein J.H. \& Ellis W.N. 1997: Climate-induced changes in the Microlepidoptera fauna of the Netherlands and the implications for nature conservation. J. Insect Conserv. 1: 73-80.

Pollard E. \& Yates T.J. 1993: Monitoring Butterflies for Ecology and Conservation. Chapman \& Hall, London, 274 pp.

Regniere J. \& Nealis V. 2002: Modelling seasonality of gypsy moth, Lymantria dispar (Lepidoptera: Lymantriidae), to evaluate probability of its persistence in novel environments. Can. Entomol. 134: 805-824.

Root T.L., Price J.T., Hall K.R., Schneider S.H., Rosenzweig C. \& Pounds J.A. 2003: Fingerprints of global warming on wild animals and plants. Nature 421: 57-60.

Roy D.B. \& Sparks T.H. 2000: Phenology of British butterflies and climate change. Global Change Biol. 6: 407-416.

Sparks T.H., Roy D.B. \& Dennis R.L.H. 2005: The influence of temperature on migration of Lepidoptera into Britain. Global Change Biol. 11: 507-514.

Stefanescu C., Peñuelas J. \& Filella I. 2003: Effects of climatic change on the phenology of butterflies in the northwest Mediterranean Basin. Global Change Biology 9: 1494-1506.

Tryjanowski P., KuźNiaK S. \& Sparks T.H. 2005: What affects the magnitude of change in first arrival dates of migrant birds? J. Ornithol. 146: 200-205.

Waring P., Townsend M. \& Lewington R. 2003: Field Guide to the Moths of Great Britain and Ireland. British Wildlife Publishing, Dorset, $432 \mathrm{pp}$.

WorwoD I.P. 1997: Detecting the effects of climate change on Lepidoptera. J. Insect Conserv. 1: 149-158.

Zhou X., Harrington R., Woiwod I.P., Perry J.N., Clark S.J. \& BALE J.S. 1996: Impact of climate change on aphid flight phenology. Aspects Appl. Biol. 45: 299-305.

Received September 29, 2005; revised and accepted December 2, 2005 